\title{
Biomonitoring the intake of garlic via urinary excretion of allyl mercapturic acid
}

\author{
H. Verhagen ${ }^{1}$, G. J. Hageman ${ }^{2}$, A-L. Rauma ${ }^{3}$, G. Versluis-de Haan ${ }^{1}$, \\ M. H. M. van Herwijnen ${ }^{2}$, J. de Groot ${ }^{2}$, R. Törrönen ${ }^{4}$ and H. Mykkänen ${ }^{4}$ \\ ${ }^{1}$ TNO Voeding, P.O. Box 360, Zeist, 3700 AJ, The Netherlands \\ ${ }^{2}$ University of Maastricht, P.O. Box 616, 6200-MD, Maastricht, The Netherlands \\ ${ }^{3}$ University of Joensuu, P.O. Box 55, Joensuu, FIN-57101, Finland \\ ${ }^{4}$ University of Kuopio, P.O. Box 1627, Kuopio, FIN-70211, Finland
}

\begin{abstract}
Allium vegetables (onions, leeks, chives) and in particular garlic have been claimed to have health-promoting potential. This study was conducted to get insight into the perspectives for monitoring the intake of garlic by a biomarker approach. Chemically, the biomarker results from exposure to $\gamma$-glutamyl- $S$-allyl-L-cysteine, which is first hydrolysed by $\gamma$-glutamine-transpeptidase resulting in the formation of $S$-allyl-L-cysteine. The latter compound is subsequently $N$-acetylated by $N$-acetyltransferase into $S$-allyl-mercapturic acid (ALMA) and excreted into urine. The mercapturic acid was measured in urine using gaschromatography with mass spectrometry. Thus the intake of garlic was determined to check the compliance of garlic intake in a placebo-controlled intervention study. Results indicate that $S$-allyl-mercapturic acid could be detected in 15 out of 16 urine samples of garlic supplement takers, indicating good compliance. In addition, the intake of garlic was also monitored in a cross-section study of vegans versus controls in Finland, in which no differences in garlic consumption nor in ALMA output were recorded between vegans and controls. These data indicate good possibilities for further studies in the field of biomarkers to investigate the putative chemopreventive effects of garlic and garlic-containing products.
\end{abstract}

Biomarker: Garlic: Urine: Allyl mercapturic acid

\section{Introduction}

Among many natural ingredients that have been claimed to have health-promoting potential (Verhagen et al. 1997) allium vegetables (garlic, onions, leeks, chives) are among the most frequently mentioned foodstuffs (Lau et al. 1990; Ernst, 1997; Orekhov \& Grunwald, 1997). In particular, garlic has been considered beneficial to humans over the centuries.

A chemical characteristic of many non-nutrients found in garlic and other allium vegetables is that they contain sulphur (Carson, 1987; Block, 1992). Several such compounds have been shown to inhibit the development of experimentally induced cancers in laboratory animals. Mechanisms underlying the cancerprotective effects of garlic are: induction of detoxicating biotransformation enzymes, inhibition of nitrate to nitrite conversion, and antibiotic properties towards Helicobacter pylori (Anonymous, 1997 for review).

There have been many epidemiological studies on the relation between intake of allium vegetables and cancer risk. Out of 35 (case-control and cohort) studies 27 have reported protective effects. However, it was concluded recently that the evidence that high garlic intakes decrease the risk of stomach cancer is, as yet, insufficient (Anonymous, 1997).

A major drawback of epidemiological studies is that it is hard to establish the actual exposure to food constituents since exposure assessment relies heavily on dietary questionaries. Another approach of assessing the exposure of humans to bioactive dietary constituents is to measure the amounts present in the diet, such as by whole diet approaches or market basket studies. However, such studies are quite complicated to perform because of organisational aspects and high costs. Moreover, only relatively few samples can be measured. In addition, epidemiological studies cannot overcome inter- and intraindividual differences in intake of bioactive substances (van Poppel et al. 1997).

Hence, if there is an alternative to these methods accounting for a total diet approach as well as providing data on an individual basis this may greatly allowing for a (adverse and beneficial) health-risk assessment of bioactive

\footnotetext{
* Corresponding author: Dr H. Verhagen: Unilever Research Vlaardingen, Unilever Health Institute, P.O. Box 114, 3130 AC, Vlaardingen, The Netherlands, fax +31 10460 5993, email hans.verhagen@unilever.com
} 
Table 1. Levels of allyl mercapturic acid in $24 \mathrm{~h}$ urine samples in placebo-controlled intervention study

\begin{tabular}{|c|c|c|}
\hline Group & Treatment & Individual values ( $\mu \mathrm{g}$ ALMA in total 24-h urine sample) \\
\hline 1 & An antioxidant mixture (vitamin $\mathrm{C}$ and $\mathrm{E}, \beta$-carotene, selenium) & $\begin{array}{l}\text { Eight samples: not detectable }{ }^{a} \\
\text { One sample positive: } 107 \mu \mathrm{g} \text { ALMA }\end{array}$ \\
\hline 2 & $\begin{array}{l}\text { An antioxidant mixture (vitamin } \mathrm{C} \text { and } \mathrm{E}, \beta \text {-carotene, selenium) } \\
\text { with } 100 \mathrm{mg} \text { garlic extract (equivalent to } 300 \mathrm{mg} \text { fresh garlic) }\end{array}$ & $\begin{array}{l}\text { One sample: not detectable } \\
\text { Seven samples positive: } 20,25,26,26,63,71,881 \mu \mathrm{g} \text { ALMA }\end{array}$ \\
\hline 3 & $100 \mathrm{mg}$ garlic extract only & $\begin{array}{l}\text { One sample: not detectable } \\
\text { Eight samples positive: } 30,36,57,62,93,102,140,251 \mu \mathrm{g} \text { ALMA }\end{array}$ \\
\hline 4 & A placebo & $\begin{array}{l}\text { Four samples: not detectable } \\
\text { One sample positive: } 505 \mu \mathrm{g} \text { ALMA }\end{array}$ \\
\hline
\end{tabular}

${ }^{a}$ Below limit of detection ( $0.01 \mu \mathrm{g}$ ALMA per ml urine).

dietary constituents. To this end, the most suitable way is apply to the philosophy: 'what goes in ... must come out'. Thus by measuring excretion the intake of a compound can be determined upon application of an established correction/calculation factor accounting for metabolism and kinetics. Regarded as such, urine is an elegant physiological integration system. This approach was chosen to check the compliance of intake of food supplements, some of which contained garlic, in a placebo-controlled intervention study. In addition, the intake of garlic was also monitored in a cross-section study of vegans versus controls in Finland.

Recently, it was reported that $S$-allyl-mercapturic acid (ALMA) is a urinary metabolite arising from garlic constituents. This opens the potential for ALMA to serve as a biomarker of exposure to garlic under normal dietary conditions. Chemically, $\gamma$-glutamyl-S-allyl-L-cysteine, a natural component of garlic is first hydrolysed by $\gamma$-glutamine-transpeptidase resulting in the formation of $S$-allyl-L-cysteine. The latter compound is subsequently $N$ acetylated by $N$-acetyltransferase into $S$-allyl-mercapturic acid and excreted into urine. This mercapturic acid can be measured in urine using gas chromatography with mass spectrometry (De Rooij et al. 1996, 1997).

\section{Materials and methods}

\section{Placebo-controlled intervention study}

A total of 101 males (aged 50-56 years) enrolled for the placebo-controlled intervention study. Samples of 24-h urine were collected in the course of a 20 -week study details of which will be published elsewhere. The volunteers were administered a daily capsule for 20 weeks containing either:

Group 1: an antioxidant mixture (vitamin $\mathrm{C}$ and $\mathrm{E}, \beta-$ carotene, selenium)

Group 2: an antioxidant mixture (vitamin $\mathrm{C}$ and $\mathrm{E}, \beta-$ carotene, selenium) with $100 \mathrm{mg}$ garlic extract (equivalent to $300 \mathrm{mg}$ fresh garlic)

Group 3: $100 \mathrm{mg}$ garlic extract only

Group 4: a placebo

For the purpose of checking the compliance in this report a distinction is made only between garlic intakes (groups 2 and 3) and others (groups 1 and 4). At the end of the 20 -week period in a subset of the study 24-h urine samples were collected.

\section{Cross-section study of Finnish vegans and controls}

In the Finnish cross-section study it was investigated whether the intake of garlic is different between vegans and matched controls. Samples of 24-h urine were collected in a study of 20 middle-aged female vegans and one male vegan who were following a strict, uncooked vegan diet (living food diet), details of which have been published elsewhere (Rauma et al. 1995).

\section{Chemical analysis}

Upon sampling aliquots of urine were frozen and transported from Maastricht, The Netherlands (placebocontrolled intervention study) or from Kuopio, Finland (cross-section study of Finnish vegans and controls) to TNO for analysis. Samples are 24-hour urine samples in which the biomarker is stable for at least 3 months when stored at $-20^{\circ} \mathrm{C}$ (De Rooij et al. 1996). All urine samples were analysed for the compound allyl mercapturic acid (ALMA; $N$-acetyl-S-allyl-L-cysteine) according to the method of De Rooij et al. (1996, 1997) with slight modifications. In short, the identity of ALMA in the urine samples was determined by gas chromatographymass spectrometry (GC-MS) using a QMD-1000. Chromatography was performed using a $30 \mathrm{~m}$ HP-5-MS (P050) capillary column of $0.25 \mathrm{~mm}$ internal diameter and $0.25 \mu \mathrm{m}$ film. Helium was used as carrier gas $(50 \mathrm{kPa}$ initial pressure). The temperature of the injector and the detector were 280 and $200^{\circ} \mathrm{C}$, respectively. The column temperature was raised from $65^{\circ} \mathrm{C}$ to $280^{\circ} \mathrm{C}$. Benzyl mercapturic acid was used as an internal standard. Limit of detection was $0.01 \mu \mathrm{g}$ ALMA/ml urine.

\section{Results \\ Placebo-controlled intervention study}

Results from the compliance assessment are given in Table 1. ALMA could be detected in 15 out of 16 urines from garlic-supplement takers, but in only two out of 14 control urines. These data are indicative of good compliance among the volunteers administered garlic in their supplements. Surprisingly, the one volunteer with no detectable ALMA in his urine reportedly took his supplements. The regular consumption of garlic via other sources was not prohibited and has presumably resulted in the two positive samples in the combined groups 1 and 4 . 
Table 2. Levels of allyl mercapturic acid in 24-h urine samples from cross-section study of Finnish vegans and controls

\begin{tabular}{lccc}
\hline & Number of volunteers & $\begin{array}{c}\text { (ALMA in total 24-h urine } \\
\text { sample }(\mu \mathrm{g})(\text { mean } \pm \mathrm{SD})\end{array}$ & $\begin{array}{c}\text { Highest value } \\
(\mu \mathrm{g} \text { per 24 h) }\end{array}$ \\
\hline Vegans & $\begin{array}{c}20 \text { females } \\
1 \text { male } \\
20 \text { females } \\
\text { Controls }\end{array}$ & $92 \pm 149^{\mathrm{a}}$ & 703 \\
& 1 male & $117 \pm 235^{\mathrm{b}}$ & 1103 \\
\hline
\end{tabular}

\footnotetext{
${ }^{a}$ Six samples below limit of detection ( $0.01 \mu \mathrm{g}$ ALMA per $\mathrm{ml}$ urine).
}

${ }^{\mathrm{b}}$ Eight samples below limit of detection (0.01 $\mu \mathrm{g}$ ALMA per ml urine).

\section{Cross-section study of Finnish vegans and controls}

ALMA could be detected in 15 out of 21 samples (vegans) and 13 out 21 samples (controls). Assuming that samples in which ALMA could not be detected contained no ALMA at all the urinary excretion of ALMA was as shown in Table 2. Differences between vegans and controls were not statistically significant $(P>0.05)$.

\section{Discussion}

Only a few methods for biomonitoring garlic have been reported in the literature. In addition to the present method, other possible biomarkers for exposure to allium species include: $N$-acetyl-S-(2-carboxypropoyl)cysteine (Jandke \& Spiteller, 1987, who also report $S$-allyl-mercapturic acid as a urinary metabolite), diallyldisulphide, diallylsulphide, dimethyldisulphide (Bartzatt et al. 1992). However, the possible application of those compounds as a biomarker for garlic intake has not been studied. In addition, Minami et al. (1989) reported on breath analysis of sulphur compounds upon consumption of garlic, but their study was not meant to be for biomonitoring purposes. In addition, the applicability of the latter method is probably rather poor since odour excretion was essentially complete after $2-3 \mathrm{~h}$.

Although ALMA results from only one of the many sulphur-containing constituents of garlic, $\gamma$-glutamyl- $S$ allyl-L-cysteine, a compound different from presumed bioactive constituents of garlic, it seems to be a good marker compound for garlic intake at least on a qualitative basis. Whether this also applies for garlic intake on a quantitative basis remains to be established, especially since the content of $\gamma$-glutamyl-S-allyl-L-cysteine in garlic is variable (Mütsch-Eckner et al. 1992). In practice, 24-h urine collection provides for a good estimate of excretion since the estimated half-life of excretion of ALMA upon consumption of garlic is approximately $6 \mathrm{~h}$ (De Rooij et al. 1996, 1997) indicating essentially complete excretion within $24 \mathrm{~h}$.

One confounder to take into account is the formation of ALMA via other routes of biotransformation; De Rooij et al. $(1996,1997)$ showed that ALMA is also formed from allyl-halides. Hence workers may elicit ALMA in urine from both occupational exposure to allyl-halides (e.g. allylchloride) and dietary exposure to garlic. In practice, few people will be exposed to allyl-halides thus rendering excretion of ALMA in urine indicative of garlic exposure.

Analytically, the mercapturic acid ALMA can best be measured in urine using gas chromatography with mass spectrometry. However, hitherto only two laboratories have reported using this method (De Rooij et al. 1996, 1997; this paper). Method validation has been described by De Rooij et al. (1996). It is cheaper to use gas chromatography and sulphur-selective detection, but then the sensitivity drops by about 10-fold (De Rooij et al. 1996), thereby rendering the biomonitoring too insensitive.

In this paper we show that the applicability of this biomarker is good for measuring the compliance of the intake of garlic-containing supplements at least on a qualitative basis. In addition, we show that the regular intake of garlic can be assessed in the free-living population. The results from the cross-section study in Finland are in line with the five-day dietary records from that study in which the consumption of onions, including garlic, varied per day as follows: mean $10 \mathrm{~g}$ (min $0-\max$ $45 \mathrm{~g})$ in the vegan group, and $9 \mathrm{~g}(\min 0-\max 40 \mathrm{~g})$ in the control group. Four vegans and nine controls reported having no garlic during the study period.

In a generic way the biomonitoring of bioactive nonnutrient dietary constituents via measurement of specific compounds in urine is a suitable alternative to dietary questionnaires to establish individual dietary exposure to food supplement ingredients under study. Further studies in the field of biomarkers in epidemiology are required to investigate the putative chemopreventive effects of garlic and garlic constituents.

\section{Acknowledgements}

De Rooij et al. $(1996,1997)$ are greatly acknowledged for introducing us to the analytical methodology and for making reference compounds available.

\section{References}

Anonymous (1997) Food, Nutrition and the Prevention of Cancer: a Global Perspective. Washington DC: World Cancer Research Fund/American Institute for Cancer Research.

Bartzatt R, Blum D \& Nagel D (1992) Isolation of garlicderived sulfur compounds in urine. Analytical Letters 25, 1217-1224.

Block E (1992) The organosulfur chemistry of the genus Allium Implications for the organic chemistry of sulfur. Angewandte Chemie International Edition in English 31, 1135-1179.

Carson JF (1987) Chemistry and biological properties of onions and garlic. Food Reviews International 3, 71-103.

De Rooij BM, Boogaard PJ, Commandeur JNM, Van Sittert NJ \& 
Vermeulen NPE (1997) Allyl mercapturic acid as urinary biomarker of human exposure to allyl chloride. Occupational and Environmental Medicine 54, 653-661.

De Rooij BM, Boogaard PJ, Rijksen DA, Commandeur JN \& Vermeulen NP (1996) Urinary excretion of $N$-acetyl- $S$-allyl-Lcysteine upon garlic consumption by human volunteers. Archives of Toxicology 70, 635-639.

Ernst E (1997) Can Allium vegetables prevent cancer? Phytomedicine 4, 79-83.

Jandke J \& Spiteller G (1987) Unusual conjugates in biological profiles originating from consumption of onions and garlic. Journal of Chromatography 421, 1-8.

Lau BHS, Tadi PP \& Tosk JM (1990) Allium sativum (garlic) and cancer prevention. Nutrition Research 10, 937-948.

Minami T, Boku T, Inada K, Morita M \& Okazaki Y (1989) Odour components of human breath after the ingestion of grated raw garlic. Journal of Food Science 54, 763-765.

Mütsch-Eckner M, Sticher O \& Meier B (1992) Reversed-phase high-performance liquid chromatography of $S$-alk(en)yl-l-cysteine derivatives in Allium sativum including the determination of (+)-S-allyl-1-cysteine and gamma-L-glutamyl-S-trans-1-propenyl-L-cysteine. Journal of Chromatography 625, 183-190.

Orekhov AN \& Grunwald J (1997) Effects of garlic on atherosclerosis. Nutrition 13, 656-663.

Rauma AL, Törrönen R, Hänninen O, Verhagen H \& Mykkänen H (1995) Antioxidant status in long-term adherents to a strict uncooked vegan diet. American Journal of Clinical Nutrition 62, 1221-1227.

Van Poppel G, Verhagen H \& Heinzow B (1997) Biomarkers in epidemiological and toxicological nutrition research. In Food Chemical Risk Analysis, pp. 87-108 [D Tennant, editor]. London: Chapman and Hall.

Verhagen H, Rompelberg CJM, Strube M, Van Poppel G \& Van Bladeren PJ (1997) Cancer prevention by dietary constituents in toxicological perspective. Journal of Environment Pathology Toxicology and Oncology 16, 343-360. 